The University of Southern Mississippi

The Aquila Digital Community

Faculty Publications

6-1-2007

\title{
Multiscale Mode Dynamics of a Tethered Membrane
}

\author{
Ras B. Pandey \\ University of Southern Mississippi, ras.pandey@usm.edu \\ Kelly L. Anderson \\ Procter \& Gamble Company \\ B. L. Farmer \\ Air Force Research Laboratory, barry.farmer@wpafb.af.mil
}

Follow this and additional works at: https://aquila.usm.edu/fac_pubs

Part of the Physics Commons

\section{Recommended Citation}

Pandey, R. B., Anderson, K. L., Farmer, B. (2007). Multiscale Mode Dynamics of a Tethered Membrane. Physical Review E, 75(6).

Available at: https://aquila.usm.edu/fac_pubs/1996

This Article is brought to you for free and open access by The Aquila Digital Community. It has been accepted for inclusion in Faculty Publications by an authorized administrator of The Aquila Digital Community. For more information, please contact Joshua.Cromwell@usm.edu. 


\title{
Multiscale mode dynamics of a tethered membrane
}

\author{
R. B. Pandey, ${ }^{1}$ Kelly L. Anderson, ${ }^{2}$ and B. L. Farmer ${ }^{3}$ \\ ${ }^{1}$ Department of Physics and Astronomy, University of Southern Mississippi, Hattiesburg, Mississippi 39406-5046, USA \\ ${ }^{2}$ Corporate Modeling and Simulation, The Procter and Gamble Company, Cincinnati, Ohio 45252, USA \\ ${ }^{3}$ Materials and Manufacturing Directorate, Air Force Research Laboratory, Wright Patterson Air Force Base, Ohio 45433, USA
}

(Received 24 July 2006; revised manuscript received 13 April 2007; published 28 June 2007)

\begin{abstract}
Stochastic dynamics of a tethered membrane with a bond-fluctuating coarse-grained Monte Carlo simulation shows, in addition to diffusion, nondiffusive behavior sensitive to the type of membrane, its size, and quality of the solvent. Motion of the membrane's center node is described by the variation of the mean-square displacement $\left(R_{n}^{2}\right)$ with time step $(t)$, i.e., $R_{n}^{2} \propto t^{2 \nu}$ with the exponent $\nu \simeq 1 / 8-1 / 6$ in the short time followed by subdiffusive power laws (i.e., $\nu \sim 1 / 5,1 / 10$ ) in the intermediate time regimes before reaching diffusion $\nu=1$. The crossover between in-plane wrinkle modes is identified from the segmental (node) motion of the membrane.
\end{abstract}

DOI: 10.1103/PhysRevE.75.061913

PACS number(s): 87.16.Dg, 02.50.Ey, 87.15.Aa, 87.15.Vv

Stochastic movements of nodes tethered together in a membrane lead to dynamic modes that cause the membrane to wrinkle and crumple [1-20]. In order to understand conformational relaxation and dynamics of the membrane it is important to probe how these local modes cooperate and compete as they propagate along the membrane surface before dissipating from its open edges. The fundamental issue is how the individual motion of a tethered node orchestrates the global motion of a membrane and vice versa. We know that the stochastic motion of a particle, the simplest representation of an object, exhibits a range of dynamics from short-to-long time asymptotic time regime, i.e., diffusion, anomalous diffusion, drift, etc. [21,22]. When particles (i.e., nodes) are tethered in a string, i.e., a polymer chain, their motion becomes more complex [23-25]. The constrained movement of a node in short time shows the Rouse dynamics and diffusion in the asymptotic time regime. The stochastic dynamics of the node (particle) of the chain in an idealized melt becomes more coordinated due to further constraints imposed by the media (surrounding chains) leading to the well-known sequence of dynamics, i.e., Rouse, reptation, post-reptation, and diffusion in appropriate time regimes in increasing order [23-25]. The dynamics of a node is further modified when it is tethered in a sheet, a model for the tethered membrane [18-20]. A tethered membrane exhibits unique conformational characteristics as it wrinkles and crumples under various physical constraints, such as quality of solvent and temperature while entropy dissipates from its open boundaries [1-20]. Using a bond-fluctuating sheet [18-20], we find unique multiscale dynamics of a tethered membrane while its conformation retains its global planar conformation.

We consider a tethered membrane, i.e., a sheet of size $L_{s}^{2}$ on a cubic lattice of size $L^{3}$ with $L=64-200$ and $L_{s}$ $=16-64$. The undistorted initial configuration of the sheet is described by a set of nodes connected by bonds each with a minimum bond length which is 2 times the lattice constant on a square grid as shown in Fig. 1. A node is represented by a unit lattice cube. The bond length can fluctuate between 2 and $\sqrt{10}$ with an exception of $\sqrt{8}$ as the node attempts to move stochastically to one of its 26 adjacent cubes (referred to as sites) as in the bond-fluctuation model for polymer chains [26,27]. Empty lattice sites represent effective solvent medium.

The interaction energy of a node is described by
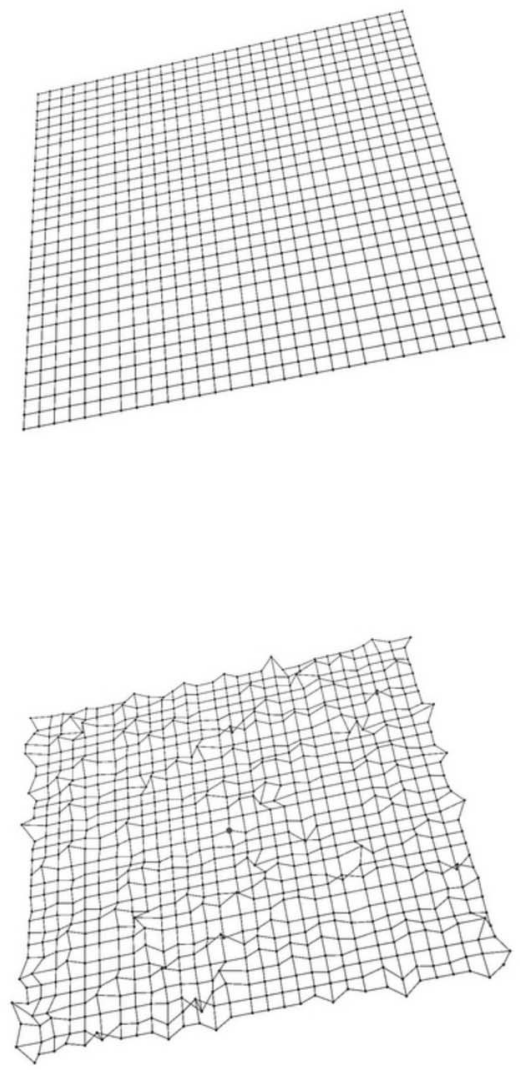

FIG. 1. Model of the membrane grid of size $30^{2}$ on a $64^{3}$ lattice. Unperturbed initial state (top) and at time step $t=5$ MCS steps (bottom) at $T=2$ with interaction $\epsilon_{n n}=-1$ to show the essence of a model with nodes, bonds, and their fluctuations. The center node is shown by the large point (bottom). 


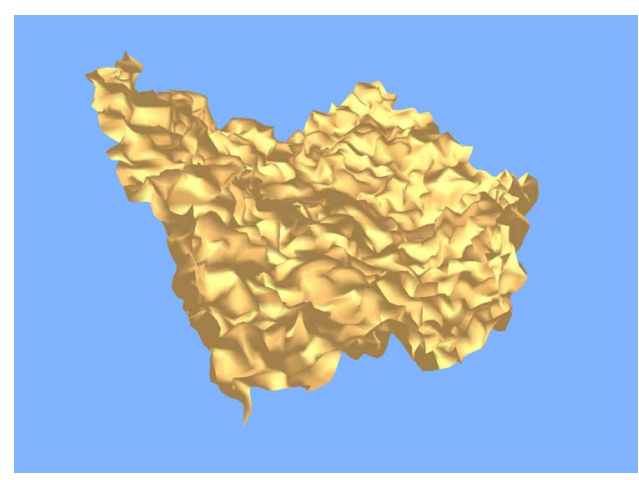

FIG. 2. (Color online) Snapshots of the membrane of size $48^{2}$ on a $150^{3}$ lattice at time step $t=2.32 \times 10^{6}$ MCS steps at $T=2$ with interaction $\epsilon_{n n}=-1$.

$$
E=\sum_{i j} J(i, j),
$$

where $i$ runs over each node and $j$ over neighboring sites within a range $r$. Different types of node-node interaction $\left[J(n, n)=\epsilon_{n n}\right]$ and node-solvent interaction $\left[J(n, s)=\epsilon_{n s}\right]$ can be considered within a range $(r)$ which can be varied $[19,20]$. We, however, are restricted here to a range of interaction $r^{2}$ up to 8 with the same interaction strength (i.e., $\epsilon_{n n}$ $=-1,0,1$ and $\left.\epsilon_{n s}=-0.5\right)$ as the effects of the variation of the range have already been studied [20]. Each node executes its stochastic move to one of its neighboring sites with the Metropolis algorithm subject to constraints on the bond fluctuation. Periodic boundary conditions are used along each direction. Attempts to move each node once define a unit Monte Carlo step (MCS) time [27]. We keep track of the meansquare displacements of the center node $\left(R_{n}^{2}\right)$ of the membrane and that of its center of mass $\left(R_{c}^{2}\right)$, and its radius of gyration $\left(R_{g}\right)$ during the simulation. Different lattice sizes $64^{3}-200^{3}$, each with many independent runs are used to test for the finite size effects and to obtain reliable averages. No finite size effect is observed on the qualitative nature of the variations of the physical quantities. Figure 2 shows a typical snapshot of the sheet at a late stage of the simulation at $T$ $=2$ with $\epsilon_{n n}=-1$. Change in the conformation of the sheet from its initial square planar configuration is clearly visible with the presence of wrinkles. The magnitudes of wrinkling and crumpling of a tethered membrane have been extensively studied including the effects of temperature and interaction (strength as well as range) as pointed out in our previous study $[19,20]$. Wrinkles are caused by the local movements of connected nodes. How the global dynamics emerge from the interplay between competition and cooperation of these dynamical modes is one of the fundamental questions that needs to be addressed. One of the unique features of a tethered membrane is the propagation of wrinkles along the connected pathways and their dissipation from the open edges, which are better seen in visual animations.

A detailed analysis of the motion of the center node is crucial in understanding the multiscale mode dynamics, i.e., from short time dynamics of a node to its asymptotic long time behavior. Let us first examine the global motion of the
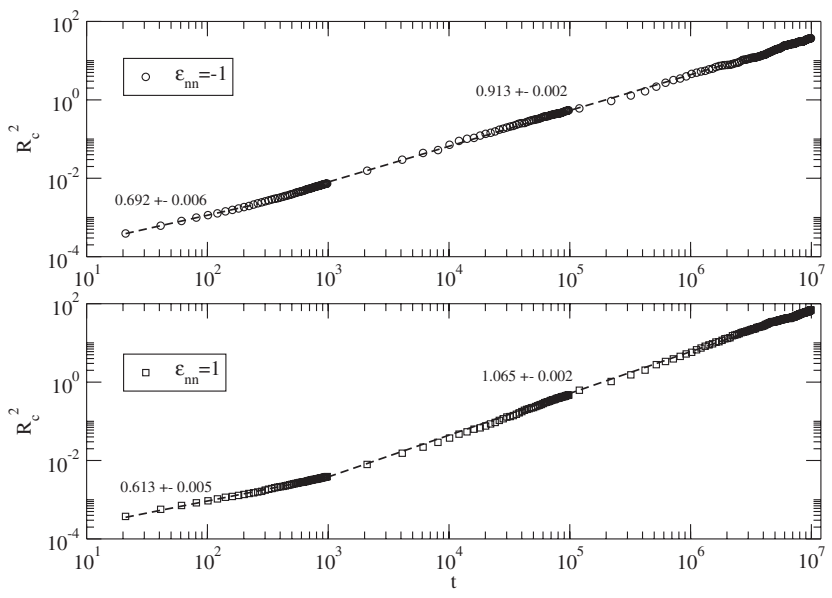

FIG. 3. Variation of mean-square displacement of the center of mass $\left(R_{c}^{2}\right)$ with the time step $t$ of the membrane of size $64^{2}$ on a $200^{3}$ lattice with interactions $\epsilon_{n n}=-1,1$ with $\epsilon_{n s}=-0.5$ at the temperature $T=2$. 10-1000 number of independent samples are used [20]. Values of slopes $(2 \nu)$ for corresponding data points are shown in all figures that follow.

membrane from the variation of the mean-square displacement of the center of mass with the time steps as shown in Fig. 3. Clearly the membrane reaches its diffusive motion soon after $10^{3}$ time steps. The relaxation time required to reach its diffusive behavior depends on temperature, interactions, molecular weight, etc. $[19,20]$. For example, it takes longer to reach asymptotic diffusive behavior at lower temperatures.

In order to probe segmental dynamics and in-plane local modes it is crucial to examine the movement of the interior nodes. Variation of the mean-square displacement $\left(R_{n}^{2}\right)$ of the center node with the time step is presented in Fig. 4. We can recognize three types of power-law dynamics in sequence: (i) short time dynamics, $R_{n}^{2} \propto t^{2 \nu_{1}}$ with $\nu_{1} \simeq 1 / 6$ followed by (ii) a faster dynamics $R_{n}^{2} \propto t^{2 \nu_{2}}$ with $\nu_{2} \simeq 1 / 3$ before slowing down substantially, $R_{n}^{2} \propto t^{2 \nu_{3}}$ with $\nu_{3} \simeq 1 / 10$. Comparison of Figs. 3 and 4 reveals that the node does not catch up with their collective, i.e., global diffusive dynamics in our observation time. The dynamics of the membrane is expected to be much more complex than that of a particle or linear chain due to multiple connected pathways for the local modes to propagate. Note that the sequence of multiscale dynamics in Fig. 3 provides a relative estimate of how the in-plane wrinkles propagate with crossover from one dynamical mode to another. Further, we see that the power-law exponents for these well-defined subdiffusive modes depend on the type of membrane $\left(\epsilon_{n n}=1,-1\right)$. We also find that the dynamics depend on the temperature and quality of the solvent $[19,20]$. The asymptotic behavior of the node appears to be subdiffusive with a very small value of the exponent $\nu_{3} \simeq 1 / 10$ even at around such long time steps $\left(10^{7}\right)$ of its stochastic moves. Obviously, the motion of the node must be diffusive, and 10 million time steps are not long enough to qualify for the asymptotic time regime in order to clearly see its Fickian diffusion. The local dynamics should eventually catch up with the global motion of the membrane.

A closer look at the long time data $\left(\sim 10^{7} \mathrm{MCS}\right)$ shows 

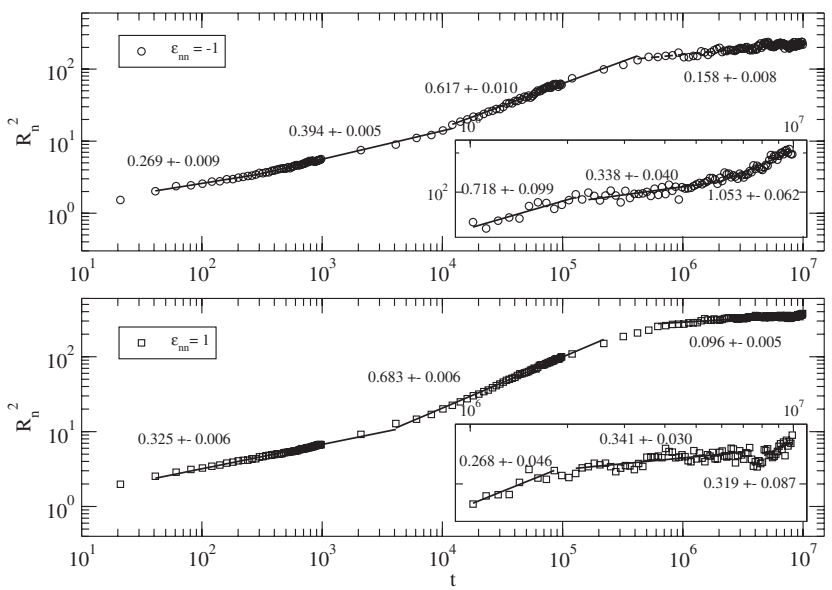

FIG. 4. Variation of mean-square displacement of the center node $\left(R_{n}^{2}\right)$ with the time step $t$ of the membrane of size $64^{2}$ on a $200^{3}$ lattice with interactions $\epsilon_{n n}=-1,1$ with $\epsilon_{n s}=-0.5$ and $T=2$. 10-1000 number of independent samples are used [20].

the onset of diffusive behavior despite large fluctuations (see the inset of Fig. 4). A large-scale simulation is performed to verify the approach to diffusive dynamics as shown in Fig. 5. Clearly, the motion of the node reaches its diffusive behavior in the last decade. The long time data also show relatively sharp crossovers consistent with Fig. 4. Trends in variations of both $R_{c}$ and $R_{n}$ further illustrate that the unified diffusive dynamics is ultimately reached as the node follows the center of mass. At such a large asymptotic scale, i.e., in the diffusive regime, the internal structure becomes irrelevant and the membrane can be treated as a particle.

Thus, we find that the local modes propagate via multiscale dynamics with well-defined crossovers. The range of different scale dynamics may depend on the type of sheet. Are the segmental dynamics independent of the sheet size? The answer is yes and no: yes as far as the general multiscale node (or mode) dynamics is concerned and no for its specific characteristics such as the crossover dynamics and relaxation. Since the entropy dissipates, the conformations remain nearly flat for most sheets (with exceptions of strong interactions, solvent, and nodes). The time required for wrinkles to propagate or dissipate from the open edges depends on the distance it travels, i.e., the size of the membrane. Figure 6 shows the variation of the mean-square displacements with the time steps, analogous to Fig. 5, for a smaller sheet $\left(32^{2}\right)$ with node-node interactions $\epsilon_{n n}=-1,0,1$. While the center of mass continues to diffuse, similar to the larger sheet, the contrast in the motion of the center node is clear (see Figs. 5 and 6). The slow mode dynamics in the intermediate time regime observed in the larger sheet (Fig. 5) is no longer seen in the smaller sheet (Fig. 6). The wrinkle nodes propagate too fast and the size of the sheet is not large enough to produce well-defined longer-lasting modes. We conjecture that the size of the sheet must be sufficiently large to observe well-defined multiscale mode dynamics. Further, the collective modes may propel the sheet faster than diffusion at times particularly in the crossover time regime, i.e., for $\epsilon_{n n}=1$ before slowing down to diffusion. Pure diffusion with $\epsilon_{n n}=0$ clarifies the differences in dynamics for different types of

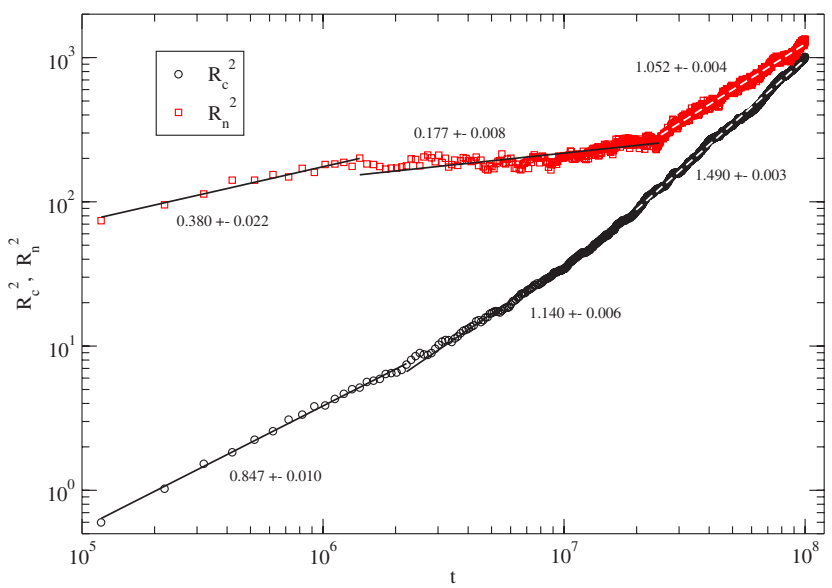

FIG. 5. (Color online) Variation of mean-square displacement of the center of mass of the membrane of size $64^{2}$ and that of its center node $\left(R_{n}^{2}\right)$ with the time step $t$ on a $200^{3}$ lattice with interactions $\epsilon_{n n}=-1$ with $\epsilon_{n s}=-0.5$ and $T=2$. 50-100 number of independent samples are used.

sheets, i.e., between a relatively more flexible $\left(\epsilon_{n n}=-1\right)$ and a stiffer $\epsilon_{n n}=1$ sheet.

These multiscale segmental dynamics, very different from those for the chain [23-25] are unique characteristics of propagating modes of a tethered membrane. A polymer chain is described by nodes tethered together via flexible bonds in a string (one-dimensional object), where each interior node is connected by two adjacent nodes. There are only two outer nodes at the ends through which it is relatively difficult for the internal modes (conformational fluctuations) to dissipate. For a free chain, the short time movement of the node is described by Rouse dynamics (crudely speaking, random walk of a node on a random walk, the constraint posed by the chain) where its rms displacement $R=C_{1} t^{1 / 4}$ followed by the diffusion $R=D t^{1 / 2}$ (in asymptotic time regime) where $C_{1}$ and $D$ are constants.

A sheet is described by nodes tethered together via flexible bonds in a plane (a two-dimensional object) where each interior node is connected by four adjacent nodes in our $L_{s}^{2}$ sheet (there can be more or less adjacent nodes depending on the type of mesh, i.e., a triangular or hexagonal mesh). There are many outer nodes on the peripheries $\left(4 L_{s}\right)$ through which it is easier for the internal modes (entropy) to dissipate. The short time movement of the interior node is described by dynamics such that its rms displacement $R=C_{2} t^{1 / 8}$ [crudely speaking random walk of a random walker (node) is severely constrained by connected adjacent nodes] with a constant $C_{2}$. This is an analog of Rouse dynamics of chain but with an exponent one-half of its size. Note that the idea of Rouse relaxation and therefore the Rouse dynamics of a chain is used in the scaling analysis of the tethered surface by Kantor et al. [1] without explicitly quantifying it. This short time dynamics, before the asymptotic diffusion, is followed by the intermediate dynamics (one may think of a constrained random walk in a different effective medium on a larger length scale) with possibly more than one power-law regime (see Figs. 4 and 5), $R=A_{2} t^{\nu_{2}}$, where $A_{2}$ is a constant and the exponent $\nu_{2}$ is subdiffusive. Thus, not only new short time 

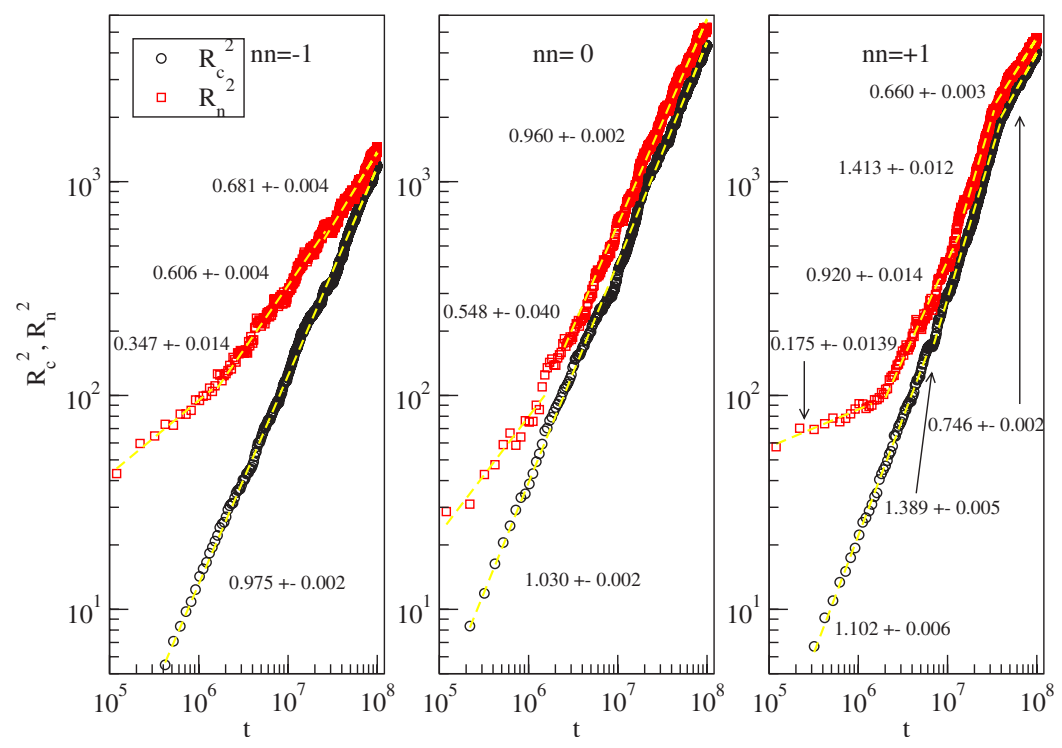

FIG. 6. (Color online) Variation of mean-square displacement of the center of mass of the membrane of size $32^{2}$ and that of its center node $\left(R_{n}^{2}\right)$ with the time step $t$ on a $100^{3}$ lattice with nodenode $(n n)$ interactions $\epsilon_{n n}$ $=-1,0,1$ with $\epsilon_{n s}=-0.5$ and $T=2$. 73-100 number of independent samples are used.

dynamics, analog of Rouse, are observed, but also the intermediate regime which is absent for a free chain. Note that it spans over the time in which the internal mode covers a distance approximately the size of the sheet; it also depends on the interaction matrix in which the sheet is embedded. In the long-time asymptotic regime the motion of the node becomes diffusive when the sheet behaves like a free particle. In passing, we would like to point out that we have already examined [19] the scaling of the radius of gyration $R_{g}$ of the sheet with its linear dimension, $R_{g} \sim L_{s}^{\gamma}$, and found that the scaling exponent $\gamma \simeq 1$, consistent with the upper bound predicted by Kantor et al. [1] for a flat sheet.

It is worth pointing out that the transient dynamics of the flat sheet is not the same as the dynamics of a crumpled sheet such as crumpled paper [29] or a thin sheet of Mylar [30]. Such crumpled sheets where the energy is presumably stored in a connected and disconnected network of ridges and cones exhibit elastic and plastic deformation responses [29,30]. Random distribution of ridges that cause long-lived scars in such crumpled objects leads to in-plane heterogeneity that may affect the propagation of entropic modes more severely than the flat unscarred sheets. The dynamics of such crumpled surfaces is very complex due to ridges and scars. Our model for sheet is too flexible to trap such local ridges [for the parameters (interaction and temperature) considered here] leading to dissipation of entropy. Wrinkles in Fig. 2 are not long-lasting ridges or scars. In other words, the temporal ridges, due to wrinkles, are the annealed in-plane disorder effect of which, on the mode dynamics (segmental dynam- ics) of the flexible sheet, are expected to be less pronounced than that in quenched disordered systems with scars.

The response (tensile or shear) properties from small scale to large scale will depend on these transient (short, intermediate, asymptotic) dynamics similar to corresponding dynamics (short and asymptotic) for a free chain. The intermediate dynamics is somewhat similar to the intermediate dynamics of a chain in a complex environment such as a melt matrix. We believe that the consequences of transients (which may be large on time scale) should be observable in viscoelastic response properties at the time scales comparable to multiscale relaxation regimes of the sheet. Let us consider a polymer chain in entangled melt. The dynamics of the center node exhibits multiple transients: (i) Rouse, (ii) reptation, (iii) post-reptation, (iv) diffusion. The observed dependence of viscosity on the molecular weight (size of the chain) is described by an approximate scaling relation which is usually explained via reptation, a transient dynamics of the chain. In the spirit of similar scaling arguments [23-25] one would expect the intermediate transient dynamics to exhibit its consequences in viscoelastic response of sheets. We hope this study will help the understanding of exfoliation of clay platelets in polymer nanocomposites [28] and identifying the segmental mode dynamics of tethered membranes [1-20] and stimulate further interest.

Support from the Materials and Manufacturing Directorate of the Air Force Research Laboratory, the Air Force Office of Scientific Research (AFOSR), and the NSF-EPSCoR is gratefully acknowledged.
[1] Y. Kantor, M. Kardar, and D. R. Nelson, Phys. Rev. Lett. 57, 791 (1986).

[2] Y. Kantor, M. Kardar, and D. R. Nelson, Phys. Rev. A 35, 3056 (1987).

[3] M. Kardar and D. R. Nelson, Phys. Rev. Lett. 58, 1289 (1987).
[4] M. Plischke and D. Boal, Phys. Rev. A 38, 4943 (1988).

[5] F. F. Abraham, W. E. Rudge, and M. Plischke, Phys. Rev. Lett. 62, 1757 (1989).

[6] Statistical Mechanics of Membranes and Interfaces, edited by D. R. Nelson, T. Piran, and S. Weinberg (World Scientific, 
Singapore, 1989).

[7] J.-S. Ho and A. Baumgartner, Phys. Rev. Lett. 63, 1324 (1989).

[8] F. F. Abraham and D. R. Nelson, J. Phys. (France) 51, 2653 (1990).

[9] R. Lipowsky, Nature (London) 349, 475 (1991).

[10] Xin Wen et al., Nature (London) 355, 426 (1992).

[11] Y. Kantor and K. Kremer, Phys. Rev. E 48, 2490 (1993).

[12] J. F. Douglas, Phys. Rev. E 54, 2677 (1996).

[13] M. Bowick and A. Travesset, Phys. Rep. 344, 255 (2001).

[14] J.-P. Kownacki and H. T. Diep, Phys. Rev. E 66, 066105 (2002).

[15] Y. Nishiyama, Phys. Rev. E 70, 016101 (2004).

[16] L. C.-L. Lin and F. L. H. Brown, Biophys. J. 86, 764 (2004).

[17] J. A. Astrom, J. Timonen, and M. Karttunen, Phys. Rev. Lett. 93, 244301 (2004).

[18] R. B. Pandey, K. L. Anderson, H. Heinz, and B. L. Farmer, J. Polym. Sci., Part B: Polym. Phys. 43, 1041 (2005).

[19] R. B. Pandey, K. L. Anderson, and B. L. Farmer, J. Polym. Sci., Part B: Polym. Phys. 43, 3478 (2005).

[20] R. B. Pandey, K. L. Anderson, and B. L. Farmer, J. Polym.
Sci., Part B: Polym. Phys. 44, 2512 (2006).

[21] R. B. Pandey and D. Stauffer, Phys. Rev. Lett. 51, 527 (1983).

[22] S. Havlin and Ben-Avraham, Adv. Phys. 36, 695 (1987).

[23] P. G. de Gennes, Scaling Concepts in Polymer Physics (Cornell University Press, Ithaca, 1979).

[24] M. Doi and S. F. Edwards, The Theory of Polymer Dynamics (Clarendon, Oxford, 1994).

[25] M. Rubinstein and R. H. Colby, Polymer Physics (Oxford University Press, Oxford, 2003).

[26] I. Carmesin and K. Kremer, Macromolecules 21, 2819 (1988).

[27] Monte Carlo and Molecular Dynamics Simulations in Polymer Science, edited by K. Binder (Oxford University Press, New York, 1995).

[28] Polymer Nanocomposites: Synthesis, Characterization, and Modeling, edited by R. Krishnamoorti and R. A. Vaia, ACS Symposium Series 804 (American Chemical Society, Washington, D.C., 2002).

[29] D. L. Blair and A. Kudrolli, Phys. Rev. Lett. 94, 166107 (2005).

[30] K. Matan, R. B. Williams, T. A. Witten, and S. R. Nagel, Phys. Rev. Lett. 88, 076101 (2002). 